\title{
A rare cause of biliary obstruction - intraductal neuroendocrine tumor of the right hepatic biliary duct: a case report
}

\author{
Mirela Danila $^{1}$, Roxana Sirli' ${ }^{1}$, Alina Popescu ${ }^{1}$, Nicoleta Iacob $^{2}$, Ana-Maria Ghiuchici ${ }^{1}$
}

${ }^{1}$ Department of Gastroenterology and Hepatology, "Victor Babeș "University of Medicine and Pharmacy, ${ }^{2}$ Neuromed Diagnostic Imaging Center Timișoara, Romania

\begin{abstract}
Primary biliary tract neuroendocrine tumors (NETs) are extremely rare tumors that account for $0.2-2 \%$ of all gastrointestinal neuroendocrine tumors. The typical presentation is with jaundice and other symptoms related to biliary obstruction. We present a case of right hepatic duct NET in a 27 -year-old female patient, asymptomatic, presented for a routine ultrasound examination that revealed moderate dilatation of the intrahepatic biliary ducts and a $20 \mathrm{~mm}$ hyperechoic lesion in the right hepatic biliary duct. Additional imaging was performed with the presumptive diagnosis of cholangiocarcinoma. After surgery, the histopathological and immunohistochemical report was conclusive for the diagnosis of G2 well-differentiated NET.

Keywords: neuroendocrine tumor; right hepatic bile duct; biliary obstruction
\end{abstract}

\section{Introduction}

Primary biliary tract neuroendocrine tumors (NETs) are extremely rare tumors with less than 100 reported cases in the literature [1,2], accounting for $0.2-2 \%$ of all gastrointestinal neuroendocrine tumors $[2,3]$. NET is derived from embryonal neural cells called Argentaffin or Kulchitsky-enterochromaffin cells that have the potential of secreting serotonin. These cells are found in the highest proportion in the small intestine and rarely within the biliary ducts, explaining the low incidence of extrahepatic biliary neuroendocrine tumors (NET) [2,4-6].

According to the World Health Organization (WHO) classification system (2010) NETs are defined as neoplasms with neuroendocrine differentiation. WHO clas-

Received 10.03.2020 Accepted 21.04.2020

Med Ultrason

2021, Vol. 23, No 2, 235-237

Corresponding author: Mirela Danilă, MD

73/9, Martir Petru Domășneanu street,

300351 Timişoara, Romania

Phone: + 40-723-480.752

Fax: + 40-256-488.003

E-mail: danila.mirela@umft.ro sifies neuroendocrine neoplasms based on their Ki67 and Mitotic indices. In 2017, the WHO classification was updated based on recent evidence [7-9].

We report a case of primary biliary NET arising from the right hepatic biliary duct. After an extensive search of the literature, no references regarding a NET of the right hepatic biliary duct was found.

\section{Case report}

A 27-year-old female patient, asymptomatic, with no known pathology, presented for the evaluation of a minor cytolytic syndrome. Clinical examination revealed no hepatomegaly/splenomegaly, jaundice or other abnormalities. Laboratory tests - slightly increased transaminases: AST $40 \mathrm{U} / \mathrm{L}(\mathrm{N}<35)$, ALT $64 \mathrm{U} / \mathrm{L}(\mathrm{N}<35)$ with negative markers of viral B or C chronic hepatitis. Diagnostic workup with an abdominal ultrasound revealed moderate dilatation of the intrahepatic biliary ducts and a 20 $\mathrm{mm}$ hyperechoic lesion in the right hepatic biliary duct (fig 1). Contrast enhanced ultrasound (CEUS) examination showed a homogenous hyperenhancement pattern in the arterial phase with intense washout in the late phase, suggesting malignancy (fig 2). 


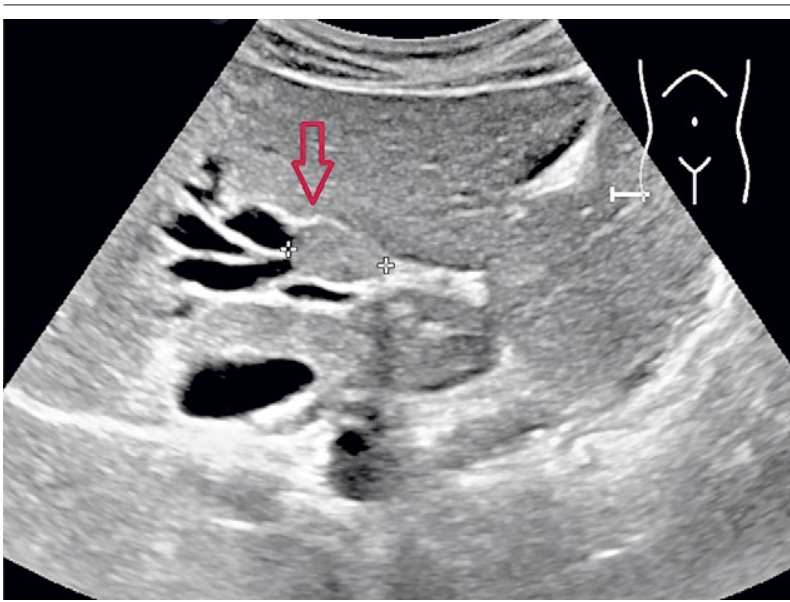

Fig 1. Abdominal ultrasound showing moderate dilatation of the intrahepatic biliary ducts and a slightly hyperechoic lesion, $20 \mathrm{~mm}$ in size, in the right hepatic biliary duct.

Additional laboratory tests showed: mild cholestasis AP $206 \mathrm{U} / \mathrm{L}(\mathrm{N}<129), \gamma$-glutamyl transpeptidase $286 \mathrm{U} / \mathrm{L}$ $(\mathrm{N}<38)$. Tumor markers, including carcinogenic embryonic antigen (CEA), carbohydrate antigen 19-9 (CA19-9) and alpha-fetoprotein, were normal.

Subsequently, abdominal MRI and MRCP confirmed a solid lesion in the right hepatic biliary duct, with discreet hyperintensity in $\mathrm{T} 2$ and restricted diffusion. In the delayed phase, the lesion presented washout, suggestive of malignancy (fig 3).

The presumptive diagnosis was biliary obstruction due to localized Klatskin/cholangiocarcinoma tumor without evidence of metastasis.

The patient was referred to the Department of Surgical Oncology and resection of the common bile duct, cholecystectomy, Roux-en-y hepatico-jejunostomy reconstruction and locoregional lymphadenectomy was performed. The histopathological and immunohistochemical report of the resected tumor was conclusive for the diagnosis of a well-differentiated neuroendocrine tumor (NET G2). Immunohistochemical staining showed that the tumor cells were strongly positive for Chromogranin A and Synaptophysin with the Ki-67 index $3-5 \%$.

After surgery, the patient received chemotherapy and has remained under oncologic surveillance. There was no recurrence of the disease at three years after surgery.

\section{Discussion}

Neuroendocrine tumors derive from Argentaffin or Kulchitsky-enterochromaffin cells. The most common sites of NET occurrence are ileum, appendix, bladder, prostate, rectum, stomach, bronchi, pancreas, and biliary tree. The paucity of enterochromaffin cells in the biliary tree explains the rare incidence of NETs in the biliary system. Biliary tract NETs are extremely rare and only one-fifth of these tumors are well-differentiated NETs $[5,10]$. Biliary tract NETs are often non-secreting tumors and larger tumors over $2 \mathrm{~cm}$ are associated with aggressive behavior [5].

Published data show that in most cases the presenting symptom was related to the local invasion of the tumor [10]. The most common presenting symptom in patients with biliary tract NETs is jaundice, followed by pain and other nonspecific symptoms (pruritus, nausea, vomiting, weight loss) $[1,11]$. Our patient was asymptomatic probably due to the small dimensions of the tumor.

Cholangiocarcinoma is the most frequent biliary tract malignancy. Differential diagnosis between cholangiocarcinoma and other bile duct tumors, such as NET, is challenging before surgical resection and histopathological exam. In our case, the final diagnosis was also established postoperatively.

NETs of the biliary ducts are generally well-differentiated, slow-growing tumors with rare metastatic spread and have a favorable prognosis with a high-rate survival, mostly in cases in which curative surgical resection is possible [11-15]. Our patient also had a well-differentiated biliary tract NET- G2.

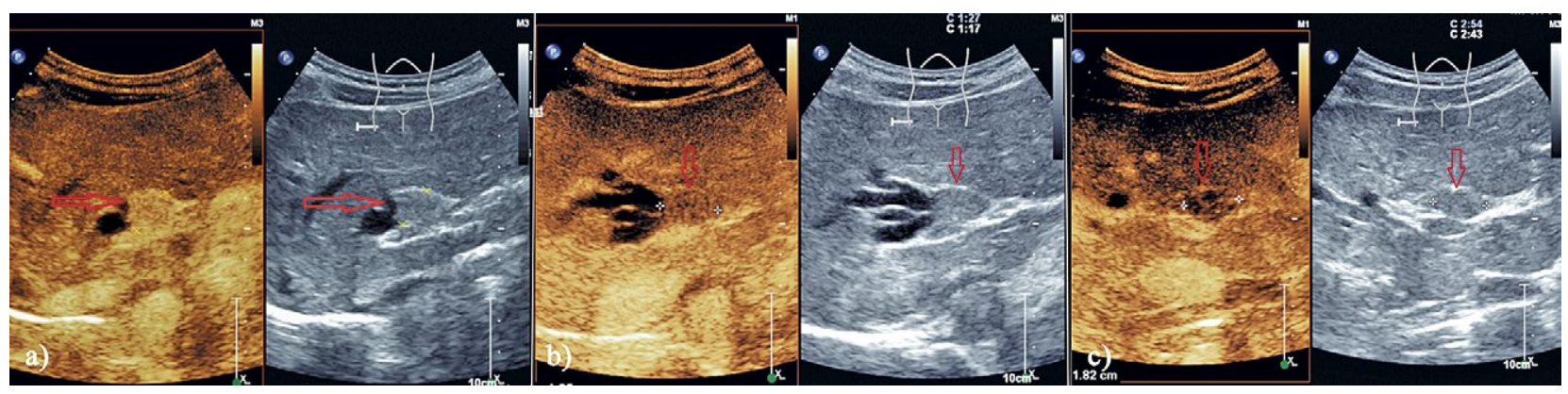

Fig 2. CEUS examination: a) homogenous hyperenhancement pattern in the arterial phase; b) mild washout starting in the portal vascular phase; c) obvious washout in the late vascular phase. 


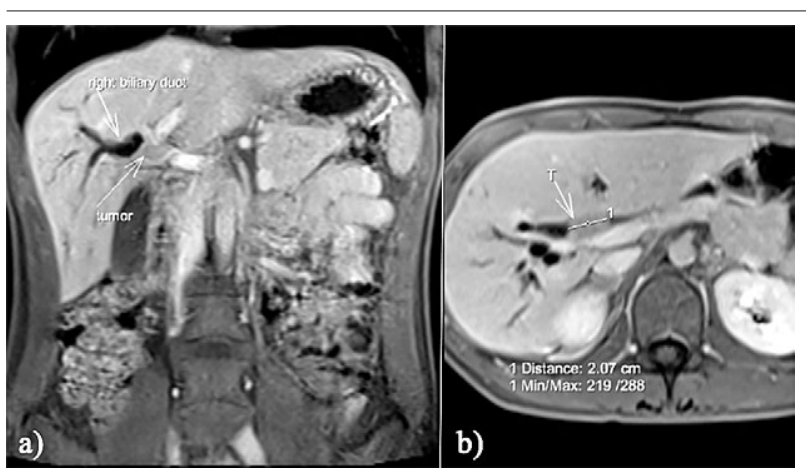

Fig 3. T1- Gadolinium postcontrast sequences, late phase: coronal (a) and axial plane (b).

The literature search did not reveal other cases of NET isolated in the right hepatic biliary duct, possibly making our case the first reported case of an intraductal NET of the right hepatic biliary duct.

In conclusion, biliary tract NETs have as a typical presentation, jaundice and other symptoms related to biliary obstruction, but patients can also be asymptomatic. Due to the absence of specific symptoms, a correct preoperative diagnosis is rare: diagnosis is usually made postoperatively, based on the histopathology exam. Radical tumor surgery is the only available curative approach, with high survival rates.

\section{References}

1. Khan FA, Stevens-Chase A, Chaudhry R, Hashmi A, Edelman D, Weaver D. Extrahepatic biliary obstruction secondary to neuroendocrine tumor of the common hepatic duct. Int J Surg Case Rep 2017;30:46-49.

2. Modlin IM, Sandor A. An analysis of 8305 cases of Carcinoid tumors. Cancer 1997;79:813-829.

3. Lauffer JM., Zhang T, Modlin IM. Review article: current status of gastrointestinal carcinoids. Aliment Pharmacol Therap 1999;13:271-287.

4. Barron-Rodriguez LP, Manivel JC, Mendez-Sanchez N, Jessurun J. Carcinoid tumors of the common bile duct: evidence for its origin in metaplastic endocrine cells. Am J Gastroenterol1991;86:1073-1076.

5. Noronha YS, Raza AS. Well-differentiated neuroendocrine (carcinoid) tumors of the extrahepatic biliary ducts. Arch Pathol Lab Med 2010;134:1075-1079.

6. Kuwabara H, Uda H. Small cell carcinoma of the gallbladder with intestinalmetaplastic epithelium. Pathol Int 1998;48:303-306.

7. Bosman FT, Carneiro F, Hruban RH, Theise ND. WHO Classification of Tumours of the Digestive System. 4th ed. Lyon: IARC, 2010.

8. Lloyd RV, Osamura RY, Klöppel G, Rosai J. WHO Classification of Tumours of Endocrine Organs. 4th ed. Lyon: IARC, 2017

9. UedaY, Toyama H, Fukumoto T, Ku Y. Prognosis of Patients with Neuroendocrine Neoplasms of the Pancreas according to the World Health Organization 2017 Classification. JOP 2017; 18 S(3):366-370.

10. Michalopoulos N, Papavramidis TS, Karayannopoulou G, Pliakos I, Papavramidis ST, Kanellos I. Neuroendocrine tumors of extrahepatic biliary tract. Pathol Oncol Res 2014;20:765-775.

11. Bhandarwar AH, Shaikh TA, Borisa AD, Palep JH, Patil AS, Manke AA. Primary neuroendocrine tumor of the left hepatic duct: a case report with review of the literature. Case Rep Surg 2012;2012:786432.

12. Nesi G, Lombardi A, Baltignani G, Ficari F, Rubio CA, Tonelli F. Well-differentiated endocrine tumor of the distal common bile duct: a case study and literature review. Virchows Arch 2006;449:104111.

13. Oskuie AE, Valizadeh N. Carcinoid tumor of the common bile duct misdiagnosed as cholangiocarcinoma. Middle East J Cancer 2011;2:139-142.

14. Costin AI, Păun I, Păun M, Constantin VD, Vârcuş F. Primary neuroendocrine tumors-an extremely rare causeof obstruction of extrahepatic bile ducts: a case report. Rom J Morphol Embryol 2017;58:641-644.

15. Choi J, Lee KJ, Kim SH, Cho MY. Preoperative diagnosis of well-differentiated neuroendocrine tumor in common hepatic duct by brush cytology: A case report. Diagn Cytopathol 2019;47:720-724. 